\title{
Effects of CEO Turnover and Board Composition Reform on Improvements in the Internal Control Quality
}

\author{
Hiroshi Uemura ${ }^{1}$ \\ ${ }^{1}$ Kochi University of Technology, Kochi, Japan \\ Correspondence: Hiroshi Uemura, Kochi University of Technology, 2-22Eikokuji Kochi, Kochi 780-8515, Japan. Tel: \\ 818-8821-7152. \\ Received: May 10, 2018 \\ Accepted: May 28, 2018 \\ Online Published: June 3, 2018 \\ doi:10.5430/ijfr.v9n3p36 \\ URL: https://doi.org/10.5430/ijfr.v9n3p36
}

The author disclosed receipt of the following financial support for the research of this article: This work was supported in part by Grant-in-Aid for Scientific from Japan Society for the Promotion of Science.

\begin{abstract}
Several serious accounting scandals have occurred in Japan in recent years (e.g., Olympus); however, the government, regulators, and auditing standard setters have struggled to identify new directions for corporate governance in listed companies, such as standard setting to address risks of fraud in an audit or the adoption of new corporate governance codes. The validity and effectiveness of monitoring by outside directors have received criticism within such a context. Nevertheless, in 2015, accounting fraud at Toshiba was discovered, which surprisingly involved upper management; the outside directors had failed to detect and prevent this fraud. Again, the monitoring function of the Japanese board of directors and outside directors was viewed with suspicion. Thus, this study examines Japanese corporations that disclose significant deficiencies (SDs) in internal controls over financial reporting (ICFR) and determines whether replacing the chief executive officer (CEO) and enhancing board members' independence and financial expertise are followed by SD remediation. The results indicate that Japanese companies that disclose SDs in ICFR are more likely to replace their CEOs and enhance board independence. In addition, this study finds that although these actions do not affect SD remediation, upgrading the board's accounting expertise does correlate positively with SD remediation. Moreover, if a company remediates a SD by increasing the number of accounting experts on the board, an increase in audit fees during the following term can be mitigated. These findings should be of interest to Japan's regulators, auditing standard setters, and financial statement users when considering improvements in the quality of internal controls. In particular, these individuals must realize that the control environment is not improved in Japanese firms merely by replacing the CEO and increasing board independence, particularly because new CEOs encounter difficulties in changing the environment established by their predecessors.
\end{abstract}

Keywords: corporate governance expertise, corporate governance independence, executive turnover, significant deficiencies, audit fees

\section{Introduction}

The Financial Instruments and Exchange Act of 2006 (J-SOX) requires that the upper management at all publicly traded Japanese companies report their assessment of the company's internal controls over financial reporting (ICFR) and present audit reports confirming the validity of their assessments (Sections 24 and 193) (Note 1). J-SOX also requires management to disclose all significant deficiencies (SDs) in ICFR at the fiscal year-end. However, after the introduction of J-SOX, major accounting scandals occurred (e.g., accounting fraud by Olympus and Toshiba), subjecting the chief executive officer's (CEO's) responsibility, the independence of corporate governance, and the effectiveness of monitoring by outside directors to severe criticism. After the scandals came to light, CEOs and boards of directors were replaced or reformed in each case. Within this context, a primary research question centered on whether replacing CEOs and reforming corporate governance in Japanese corporations affected the quality of financial reporting. This study examines the relation between the disclosure of SDs in ICFR and a corporation's CEO and the independence of its board of directors.

In the U.S., CEOs' responsibility for financial reporting increased after the passage of the Sarbanes-Oxley Act in 
2002 (U.S.-SOX) (Feldmann, Read, \& Abdolmohammadi, 2009), which accelerated the movement toward more independent boards (Linck, Netter, \& Yang, 2008). Coates \& Srinivasan (2014) argue that J-SOX is contained provisions equivalent to those in both section 302 and 404 of U.S.-SOX, and Eight Directive on securities disclosure that European Union adopted largely tracked much of the contents of U.S.-SOX. Moreover, U.K. law (the Companies Act of 2004) requires firms listed Main market of the London Stock Exchange to report whether firms can comply with Listing Rules that include voluntary application of the Turnbull Guidance on internal control on the Combined Code. Listed firms can comply by disclosing or by explaining why they do not comply. A core element of the Combined Code is its recommendation to compose at least half the board of independent non-executives. Another is the separation of the positions of board chairman and CEO (see, Hopt, \& Leyens, 2004). On the other hand, German adopts the tow-tier corporate governance system. In the two-tier system, the management board is responsible for running the firm and a supervisory board that is responsible for the appointment and oversight of the management board (see, Ghoshray, 2004).

Although the proportion of outside directors on the boards of publicly traded Japanese companies rose every year from 2005 to 2010 (Miyajima \& Ogawa, 2012), serious accounting scandals were frequent despite the J-SOX regulations. In such cases, the upper management was personally involved in the fraud (e.g., Olympus and Toshiba), which indicates that corporate governance monitoring may not be comprehensive. These incidents sparked public reservations regarding the internal monitoring at Japanese companies, and the Japanese Legislative Council of the Ministry of Justice reconsidered legal provisions concerning independent corporate governance because it reasoned that addition independence may restrain reckless managers (Note2).

Disclosing SDs in ICFR entails serious consequences; for example, SDs inspire less confidence in equity markets (Beneish, Billings, \& Hodder, 2008; Hammersley, Myers, \& Shakespeare, 2008). To mitigate these consequences, firms must remediate their SDs immediately, perhaps through drastic steps, such as replacing the CEO and changing the composition of the board of directors (e.g., Desai, Hogan, \& Wilkins, 2006; Hennes, Leone, \& Miller, 2008; Agrawal \& Cooper, 2014). However, replacing the CEO in Japanese firms does not necessarily improve the control environment created through management policies, ethical values, and organizational culture because former CEOs hold entrenched power and authority after leaving their positions. Kawamoto (2009) and Tanikawa (2016) noted that there is a certain tendency in the promotion system of Japanese corporations for executives to be promoted internally. Moreover, in many corporations, the CEO and chairman of the board are the same person; thus, authority is concentrated in the CEO. For example, Japanese corporations normally fill upper management positions by promoting from within, which occurred for 97\% of Japanese CEOs in 2013 (Note 3), and the former CEO selected a new CEO through his authority (Japanese Association of Corporate Directors, 2003) (Note 4). In addition, the current CEO serves as chairman of the board at $78.5 \%$ of the companies listed on the Tokyo Stock Exchange (TSE) (Tokyo Stock Exchange, 2013) (Note 5), and CEOs often remain as the board chairman after leaving office. Furthermore, certain companies designate their presidents as chairmen of the board, although presidents are generally previous CEOs (Note 6). Dong, Girardone, \& Kuo (2016) examined the relationship between national culture and corporate governance structure based on Williamson's (2000) theoretical framework of the causal chain for formative processes of corporate governance, and they found that national culture affects incentives and choices in corporate governance. Many studies in which U.S. companies are used as the sample reveal that specific characteristics (independence) affect the quality of internal controls over financial reporting. However, if the shape of corporate governance depends on the national culture, then the results may differ from those of the previous U.S. studies. If so, CEO replacement and corporate governance reform may not have a positive effect on the mindset of an organization.

\section{Literature Review and Hypothesis Development}

\subsection{Disclosure of Significant Deficiencies and Replacing the CEO}

Many studies have suggested that financial performance (e.g., price earnings ratio, sales growth, return on asset) is the trigger for CEO turnover (Kang \& Shivasani, 1995, 1996; Kaplan, 1994; Sheard, 1994), which means that negative consequences for corporations promote changes in upper management, and disclosing a SD destabilizes corporate governance and motivates firms to change upper management (Johnstone, Li, \& Rupley, 2011). Agrawal \& Cooper (2014) examine how accounting scandals lead to changes in corporate governance, particularly the replacement of the CEO, chief financial officer (CFO), and external auditor. Desai, Hogan, \& Wilkins (2006) find that financial restatements relate positively to executive dismissals (Note 7). Moreover, studies have found that the disclosure of existing SDs is positively associated with CEO replacement (Geiger \& Taylor, 2003; Johnstone, Li, \& Rupley, 2011; Marden, Edwards, \& Stout, 2003).

A material weakness is defined as a deficiency or a combination of deficiencies in ICFR such that there is a 
reasonable possibility that a material misstatement of a company's annual or interim financial statements will not be prevented or detected on a timely basis (Public Company Accounting Oversight Board: PCAOB, 2007, AS5 Appendix A-7). According to this definition, it is clear that ICFR quality affects the credibility of financial statements because SDs in ICFR increase the risk of material misstatements in financial statements. In fact, many previous studies indicate that SD disclosures damage a company's image in equity markets (Beneish, Billings, \& Hodder, 2008; Hammersley, Myers, \& Shakespeare, 2008), trigger negative market reactions (De Franco, Guan, \& Lu, 2005; Hammersley, Myers, \& Shakespeare, 2008), raise the cost of capital (Ashbaugh-Skaife, Collins, Kinney Jr., \& Lafond, 2009; Ogneva, Subramanyam, \& Raghunandan, 2007) and control risk assessments by external auditors (Hoitash, Hoitash, \& Bedard, 2008; Krishnan, Rama, \& Zhang, 2008; Raghunandan \& Rama, 2006) (Note 8). In addition, SDs indicate upper management's failure in its responsibility to design and operate effective ICFR, which in turn impacts the credibility of a firm's financial statements after Global Economic Crisis. The PCAOB discusses the potential negative impact of the economic crisis on internal controls in a Staff Audit Practice Alert (PCAOB, 2008). To maintain effective ICFR, it is necessary to invest human resource appropriately in the implementation of ICFR. If companies are eliminating staffs with internal control responsibilities, control strength may be deteriorating (Choi, Choi, Hogan, and Lee, 2013). Choi, Choi, Hogan, \& Lee (2013) find that the proportion of internal control personnel (the ratio of the number of employees involved with the implementation of internal controls) is negatively associated with the disclosure of SDs.

In Summary, because disclosing SDs seriously damages firms, decreases the reliability of financial statements, and decreases CEO reputations, it is difficult for CEOs to maintain their positions. These arguments lead to the following hypothesis.

Hypothesis 1a. Disclosure of a SD in internal controls is positively associated with the replacement of the CEO.

\subsection{Disclosure of Significant Deficiencies and Reforming Corporate Governance}

The disclosure of SDs also leads to a change in board composition. The board of directors monitors and assesses the validity of upper management's work and performance. When monitoring, if one of the components of internal control reaches a workable level and SDs are prevented or detected through the monitoring process, then the SDs are improved on a timely basis. Krishnan (2005) and Hoitash, Hoitash, and Bedard (2009) find that the quality of audit committee expertise is significantly negatively correlated with the disclosure of material weaknesses. In addition, Agrawal and Chadha (2005) find that the probability of restatement is lower in companies whose boards or audit committees have an independent director with financial expertise. Based on these results, shareholders of firms with SDs may claim that improving the independence and expertise of corporate governance improves the quality of ICFR. This argument leads to the following hypotheses.

Hypothesis 1b. Disclosure of a SD in internal controls is positively associated with the independence of the board of directors.

Hypothesis 1c. Disclosure of a SD in internal controls is positively associated with the expertise of the board of directors.

\section{Replacing the CEO or Reforming Corporate Governance and Remediating Significant Deficiencies}

Hammersley, Myers, \& Zhou (2012) focus on consecutive SD disclosures and examine the factors that interfere with remediating them the second time they occur by employing both CEO and CFO removal as factors in addressing deficiencies. As Johnstone, Li, \& Rupley (2011) also find, replacing the CEO or CFO does not significantly correlate with remediation (Note 9). However, these researchers do find that appointing a CFO with stronger accounting expertise and greater CFO-specific experience and appointing a new CEO with a superior reputation are positively associated with remediation (Note 10). In any case, the results from previous studies examining the relation between SD remediation and CEO turnover are mixed.

As discussed previously, Japanese corporate culture is very unique (Ahmadjian \& Robbins, 2001; Ouchi, 1981), and even if a CEO is replaced after the disclosure of a serious problem, the former CEO's power, management policies, and ethical values may persist. The Japanese Association of Corporate Directors (2003) conducted a questionnaire survey to gain an understanding of the role, reward, and elective dismissal of CEOs. The results indicate that many CEOs take up posts as chairmen after relinquishing their roles as CEOs (60\% and above), and the last CEO or former CEO decides on a new CEO at one's direction (60\% and above). Under this situation, it is not difficult to see that the control environment does not change easily and immediately. In fact, in the case of Toshiba, the previous three CEOs were continuously involved in the fraud, which means that great authority is given to the CEO in Japan. Under this situation, how is corporate governance designed at Japanese companies? Dong, Girardone, and Kuo (2016) suggest that corporate governance mechanisms of U.S. companies are determined by equity markets. However, Japanese firms depend on institutional regulation to govern corporations (Hansmann \& Kraakman, 2001) (Note 11). Cohen, Krishnamoorthy, \& Wright (2008) suggests that the institutional theory perspective implies that governance entails a 
ceremonial role that includes structures and processes that are important for symbolism as opposed to substantive purpose (Note 12). If Japanese corporations decide to arrange the independence of corporate governance based on this perspective, outside directors might have only a symbolic role.

However, Fama (1980) argues that the effort level of outside directors depends on competition among the team and the degree of maturity of the labor market. If the labor market for outside directors sufficiently reaches maturity and the market valuation of outside directors depends on their performance as monitors, then the effort level of outside directors increases and the effort is efficient. Abe \& Oguro (2004) suggest that in Japan, a mutual monitoring system among team members rather than a monitoring system essential to monitoring by outside directors because the labor market for outside directors has not developed adequately. The above suggestions led to the expectation that the independence of the board of directors cannot affect the quality of ICFR.

Conversely, internal directors as experts may produce results that differ from those of outside directors. Burt (2016) finds that employees are likely to share more negative information on the ICFR with internal auditors than with external auditors. Japanese firms have a strong preference for collective responsibility and accountability, and they also have a culture defined by a strong groupthink mentality (Mroczkowski \& Hanaoka, 1989). These arguments mean that employees report negative information on the effectiveness of ICFR to internal personnel or group members instead of to outside personnel. Moreover, if internal personnel have suitable responsibility and expertise, then information on the design and operation of the ICFR will gathered from employees from the bottom up, and SDs will be remediated at the right time. Krishnan (2005) and Hoitash, Hoitash, \& Bedard (2009) indicate that the expertise of audit committees affects ICFR quality, and Masulis \& Mobbs (2011) find that firms with inside directors holding outside directorships have better operating performance, particularly when monitoring is more difficult. Furthermore, Mobbs (2013) suggests that certain inside directors strengthen board monitoring, and this result persists when accounting for the endogenous firm selection of talented inside directors. Studies also find that accounting expertise is positively associated with SD remediation (Hammersley, Myers, \& Zhou, 2012; Johnstone, Li, \& Rupley, 2011).

The next hypotheses to test these propositions are as follows.

Hypothesis 2a. Replacing the CEO after the disclosure of a SD is associated with its remediation.

Hypothesis $2 \mathrm{~b}$. Improving the independence and expertise of corporate governance is positively associated with SD remediation.

\section{Methodology}

\subsection{Research Models}

The following model testing of Hypothesis 1a is based on Johnstone, Li, \& Rupley (2011), Desai, Hogan, \& Wilkins (2006), Hennes, Leone, \& Miller (2008), and Chou \& Wang (2010):

$$
\begin{aligned}
\text { Toit }+1 & =\beta 0+\beta 1 \text { SDit }+\beta 2 \text { RISTATEMENTit }+\beta 3 \text { M } \& \text { Ait }+\beta 4 \text { GCit }+\beta 5 \text { ROAit }+\beta 6 C F O / \text { Ait } \\
& +\beta \text { GROWTHit }+\beta \text { SDEBTit }+\beta \text { LLNSIZEit }+\beta 10 \text { MTBit }+\beta 11 \text { BOARDSIZEit } \\
& +\beta 12 \text { OFFICEit }+\beta 13 \text { OUTSIDEit }+\beta 14 \text { EXPERTit }+\beta 15 \text { DIRECOWNit } \\
& +\beta 16 \text { BIGSHAREit }+\beta 1 \text { FOREIGNOWNit }+\beta 18 \text { BANKINGit }+\beta 19 \text { TRUSTit } \\
& +\beta 2 \text { INDUSTRYit }+\beta 21 \text { Year Dummyi }+\varepsilon .
\end{aligned}
$$

The dependent variable (TOit+1) is an indicator variable that is equal to 1 if the CEO changes between year $t$ and $t+$ 1 and 0 otherwise (Note 13). Disclosure of a significant deficiency in year t (SDit) is an independent variable (Note 14). SD is expected to correlate positively with TO after controlling for other variables, thereby supporting Hypothesis 1a.

The model developed to test Hypotheses $1 \mathrm{~b}$ and 1c is based on Linck, Netter, \& Yang. (2008) and Boone, Field, Karpoff, \& Raheja (2007). The following model (2) tests Hypotheses 1b and 1c:

(Board Composition) it $+1=\beta 0+\beta 1$ SDit $+\beta 2$ RISTATEMENTit $+\beta 3 \mathrm{M} \& \mathrm{Ait}+\beta 4 \mathrm{GCit}+\beta 5 \mathrm{ROAit}+\beta 6 \mathrm{CFO} / \mathrm{Ait}$

$+\beta$ 7GROWTHit $+\beta 8$ DEBTit $+\beta$ LLNSIZEit $+\beta 10$ MTBit $+\beta 11$ BOARDSIZEit

$+\beta 12$ OFFICEit $+\beta 13$ DIRECOWNit $+\beta 14$ BIGSHAREit $+\beta 15$ FOREIGNOWNit

$+\beta 16$ BANKINGit+ $\beta 17$ TRUSTit $+\beta 18$ R\&Dit+ $\beta 19$ INDUSTRYit

$+\beta 20$ Year Dummyi+e. 
Model (3) and (4) test Hypotheses 2a and 2b using SD remediation (REMEDIATE ${ }_{\mathrm{it}}$ ) as a dependent variable and TO as an independent variable and is based on Bedard, Hoitash, Hoitash, \& Westermann (2012) and Hammersley, Myers, $\&$ Zhou (2012). If TO correlates positively with REMEDIATE, replacing the CEO affects SD remediation.

$$
\begin{aligned}
\text { REMEDIATEit }+1 & =\beta 0+\beta 1 \text { TOit }+\beta 2 \text { ENVIRONMWit }+\beta 3 \Delta \text { LNSIZEit }+\beta 4 \Delta \text { LNSIZEit }+\beta 5 \Delta \text { LNSEGMENTSit } \\
& +\beta 6 \Delta \text { FOREIGNSALEit }+\beta 7 \text { M } \& \text { Ait }+\beta 8 \Delta \text { MTBit }+\beta 9 \Delta \text { GROWTHit }+\beta 10 \Delta \text { ROAit } \\
& +\beta 11 \Delta \text { CFO } / \text { Ait }+\beta 12 \text { GCit }+\beta 13 \text { BIG4it }+\beta 14 \Delta \text { AUDNUMBERit }+\beta 15 \Delta \text { OUTSIDEit } \\
& +\beta 16 \Delta \text { EXPERTit }+\beta 17 \text { DIRECOWNit }+\beta 18 \Delta \text { BIGSHAREit }+\beta 19 \Delta \text { FOREIGNOWNit } \\
& +\beta 20 \Delta \text { BANKINGit }+\beta 21 \Delta \text { TRUSTit }+\beta 22 \Delta \text { INDUSTRYit }+\varepsilon .
\end{aligned}
$$

Model (3) includes variables for changes in board expertise and independence ( $\triangle$ EXPERT and $\triangle$ OUTSIDE) to test $2 \mathrm{a}$ and $2 \mathrm{~b}$. Corporations with strong corporate governance structures are known for higher quality internal controls (Goh, 2009; Hoitash, Hoitash, \& Bedard, 2009; Krishnan, 2005) (Note 15). However, this study also expects that the results will differ from those of previous studies. Moreover, Model (4) includes variables for the interaction between TO and $\triangle$ OUTSIDE and TO and $\triangle$ EXPERT as independent variables.

\subsection{Sample and Data}

Table 1 (Panel A) describes the sample selection procedure.

Table 1. Panel A: Sample selection procedure

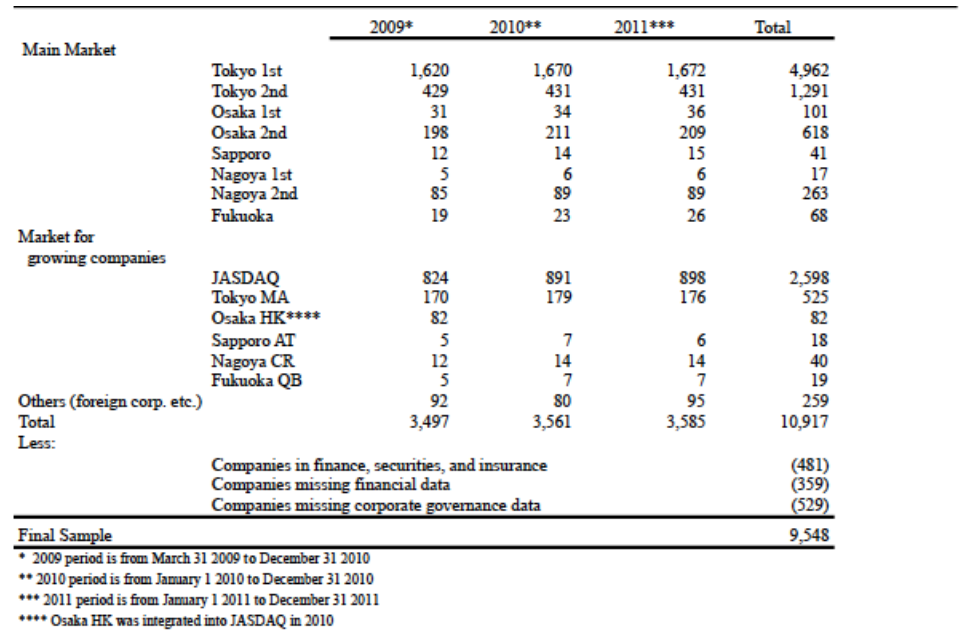

Our investigation documented 10,917 firm-year observations of publicly traded Japanese companies that disclosed management reports for fiscal years between March 31, 2009, and December 31, 2011 (Note 16). After excluding 481 observations for companies in the finance, securities, insurance, and other industries because their financial statements differ markedly from most other companies, with 359 observations excluded because there were missing financial data (Note 17) and 529 observations excluded for foreign firms and firms with no corporate governance information, the final sample contains 9,458 firm-year observations. Financial data are derived from NEEDS Financial QUEST. Data related to management's internal control reports, audit reports, and the number of business segments are derived from EDINET. Data for CEO replacements and boards of directors are derived from Directors' Quarterly Journals (Toyo Keizai Shinposya).

Table 1 (Panel B) reports the number of corporations that disclosed SDs during the sample period. In 2009, 117 corporations (3.34\%) disclosed SDs (Note 18) and 15 could not report the results of their management's assessment. In 2010, 58 corporations (1.62\%) disclosed SDs, seven issued disclaimers, and 31 companies disclosed SDs for the second consecutive year. 
Table 1. Panel B: SD disclosure

\begin{tabular}{lcccccccc} 
& 2009 & $\begin{array}{c}\text { \% of listed } \\
\text { companies }\end{array}$ & 2010 & $\begin{array}{c}\text { \% of listed } \\
\text { companies }\end{array}$ & 2011 & $\begin{array}{c}\text { \% of listed } \\
\text { companies }\end{array}$ & Total & $\begin{array}{c}\text { \% of listed } \\
\text { companies }\end{array}$ \\
\hline SD disclosure & 117 & $3.34 \%$ & 58 & $1.62 \%$ & 27 & $0.75 \%$ & 202 & $1.85 \%$ \\
Disclaimer & 15 & $0.42 \%$ & 6 & $0.16 \%$ & 4 & $0.11 \%$ & 25 & $0.22 \%$ \\
Two-year consecutive disclosure & & & 31 & $0.87 \%$ & 7 & $0.19 \%$ & 38 & $0.34 \%$ \\
Three-year consecutive disclosure & & & & & 11 & $0.30 \%$ & 11 & $0.10 \%$ \\
\hline
\end{tabular}

Table 1 (Panel C) lists the markets on which companies that disclosed SDs or issued disclaimers are listed and reports their Nikkei industry middle classification codes (Panel D). Several companies that disclosed SDs trade on an emergent market (e.g., Tokyo Mothers). Service, energy, and financial services companies had the highest rate of SD disclosures in 2009 (Note 19).

Table 1. Panel C: Number of SD disclosure firms by stock market

\begin{tabular}{|c|c|c|c|c|c|c|c|c|c|c|c|c|c|}
\hline \multirow[b]{3}{*}{ Main market } & & \multicolumn{4}{|c|}{2009} & \multicolumn{4}{|c|}{2010} & \multicolumn{4}{|c|}{2011} \\
\hline & & \multirow[b]{2}{*}{ SD dixclosure } & \multirow[b]{2}{*}{ Disclaner } & \multirow[b]{2}{*}{ Tonal } & \multirow{2}{*}{$\begin{array}{l}\text { Soflivated } \\
\text { campais }\end{array}$} & \multirow[b]{2}{*}{ SD disclosure } & \multirow[b]{2}{*}{ Discher } & \multirow[b]{2}{*}{ Total } & \multirow{2}{*}{$\begin{array}{l}\text { Yof listade } \\
\text { campunes }\end{array}$} & \multirow[b]{2}{*}{ SD disclosure } & \multirow[b]{2}{*}{ Disclamer } & \multirow[b]{2}{*}{ Total } & \multirow{2}{*}{ 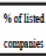 } \\
\hline & & & & & & & & & & & & & \\
\hline & Tolyo 1 1st & 28 & 2 & 30 & $1.85 \%$ & 11 & 0 & 11 & $0.65 \%$ & 3 & 0 & 3 & $0.17 \%$ \\
\hline & Tolyo 2nd & 10 & 1 & 11 & $256 \%$ & 3 & 0 & 3 & $0.69 \%$ & 2 & 0 & 2 & $0.46 \%$ \\
\hline & Osaka 1 st & 3 & 0 & 3 & $9.60 \%$ & 2 & 0 & 2 & $6.45 \%$ & 1 & 0 & 1 & $277 \%$ \\
\hline & Osaka 2nd & 9 & 0 & 9 & $4,54 \%$ & 4 & 0 & 4 & $1.89 \%$ & 3 & 0 & 3 & $143 \%$ \\
\hline & Sapporo & 0 & 0 & 0 & $0.00 \%$ & 0 & 0 & 0 & $0.00 \%$ & 0 & 0 & 0 & $0.00 \%$ \\
\hline & Nagoya lst & 0 & 0 & 0 & $0.00 \%$ & 0 & 0 & 0 & $0.00 \%$ & 0 & 0 & 0 & $0.00 \%$ \\
\hline & Nagoya 2nd & 0 & 0 & 0 & $0.00 \%$ & 0 & 0 & 0 & $0.00 \%$ & 0 & 0 & 0 & $0.00 \%$ \\
\hline & Fuluoka & 0 & 0 & 0 & $0.00 \%$ & 0 & 0 & 0 & $0.00 \%$ & 0 & 0 & 0 & $0.00 \%$ \\
\hline \multicolumn{14}{|c|}{$\begin{array}{l}\text { Market for } \\
\text { groving compamies }\end{array}$} \\
\hline & JASDAQ & 39 & 5 & 44 & $5.33 \%$ & 23 & 2 & 25 & $285 \%$ & 8 & 2 & 10 & $1.11 \%$ \\
\hline & Tolyo MA & 10 & 3 & 13 & $7.64 \%$ & 11 & 2 & 13 & $738 \%$ & 4 & 2 & 6 & $3.40 \%$ \\
\hline & Osaka HK & 10 & 1 & 11 & $13.4 \%$ & & & & & & & & \\
\hline & Sapporo AT & 2 & 2 & 4 & $80.00 \%$ & 1 & 1 & 2 & $28.57 \%$ & 0 & 0 & 0 & $0.00 \%$ \\
\hline & Nagoya $C R$ & 5 & 1 & 6 & $50.00 \%$ & 3 & 1 & 4 & $28.57 \%$ & 6 & 0 & 6 & $42.85 \%$ \\
\hline & Fuluoka QB & 1 & 0 & 1 & $20.00 \%$ & 0 & 0 & 0 & $0.00 \%$ & 0 & 0 & 0 & $0.00 \%$ \\
\hline & Total & 117 & 15 & 132 & $3.77 \%$ & 58 & 6 & 64 & $1.79 \%$ & 27 & 4 & 31 & $0.86 \%$ \\
\hline
\end{tabular}

Table 1. Panel D: Number of disclosure firms by industry type*

\begin{tabular}{|c|c|c|c|c|c|c|c|c|c|c|c|c|}
\hline & \multicolumn{4}{|c|}{2009} & \multicolumn{4}{|c|}{2010} & \multicolumn{4}{|c|}{2011} \\
\hline & SD Aichobise & Disclums & Tat & 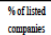 & SD ditchere & Dischere & Tond & Yof lisited & SD ditcherse & Discluer & Tot & 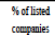 \\
\hline Electronics & 8 & 1 & 9 & $269 \%$ & 5 & 0 & 5 & $1.41 \%$ & 4 & 0 & 4 & $1.46 \%$ \\
\hline Machine & 4 & 1 & 5 & $211 \%$ & 1 & 1 & 2 & $0.89 \%$ & 1 & 0 & 1 & $0.43 \%$ \\
\hline Precision Equipment & 0 & 1 & 1 & $181 \%$ & 0 & 0 & 0 & $0.00 \%$ & 0 & 1 & 1 & $200 \%$ \\
\hline Auto Equipment & 4 & $i$ & 5 & $416 \%$ & 2 & 1 & 3 & $2.72 \%$ & 1 & 1 & 2 & $198 \%$ \\
\hline Cheminal & 5 & 0 & 5 & $1.77 \%$ & 2 & 0 & 2 & $0.96 \%$ & 1 & 0 & 1 & $0.46 \%$ \\
\hline Nouffrous Metal & 3 & 0 & 3 & $5.76 \%$ & 0 & 0 & 0 & $0.00 \%$ & 0 & 0 & 0 & $0.00 \%$ \\
\hline Ceramic & 3 & 0 & 3 & $434 \%$ & 2 & 0 & 2 & $322 \%$ & 0 & 0 & & $0.00 \%$ \\
\hline Pulp & 2 & 0 & 2 & $7.69 \%$ & 0 & 0 & 0 & $0.00 \%$ & 0 & 0 & 0 & $0.00 \%$ \\
\hline Fiber & 0 & 1 & 1 & $090 \%$ & 0 & 1 & 1 & $0.81 \%$ & 0 & 0 & 0 & $0.00 \%$ \\
\hline $\mathrm{Gm}$ & 0 & 0 & 0 & $0.00 \%$ & 0 & 0 & 0 & $0.00 \%$ & 0 & 0 & 0 & $0.00 \%$ \\
\hline Other Masuffacture & 5 & 1 & 6 & $550 \%$ & 2 & 0 & 2 & $1.78 \%$ & 0 & 1 & 1 & $0.92 \%$ \\
\hline Constraction & 9 & 0 & 9 & $4.18 \%$ & 4 & 0 & 4 & $2.19 \%$ & 2 & 0 & 2 & $1.14 \%$ \\
\hline Medicine & 1 & 0 & 1 & $175 \%$ & 2 & 0 & 2 & $3.44 \%$ & 0 & 0 & 0 & $0.00 \%$ \\
\hline Food & 7 & 2 & 9 & $7.43 \%$ & 4 & 1 & 5 & $391 \%$ & 1 & 0 & 1 & $0.76 \%$ \\
\hline Geenerl Trading & 18 & 1 & 19 & $597 \%$ & 9 & 1 & 10 & $2.77 \%$ & 4 & 0 & 4 & $1.16 \%$ \\
\hline & 8 & 1 & 9 & $280 \%$ & 4 & 1 & 5 & $135 \%$ & 3 & 1 & 4 & $1.15 \%$ \\
\hline Telecommunication & 5 & 0 & 5 & $153 \%$ & 5 & 0 & 5 & $1.43 \%$ & 3 & 0 & 3 & $0.85 \%$ \\
\hline Service & 22 & 2 & 24 & $802 \%$ & 11 & 0 & 11 & $4.77 \%$ & 5 & 0 & 5 & $139 \%$ \\
\hline Real Estate & 5 & 1 & 6 & $594 \%$ & 1 & 0 & 1 & $1.02 \%$ & 1 & 0 & 1 & $0.92 \%$ \\
\hline Transportation & 4 & 0 & 4 & $6.66 \%$ & i & 0 & 2 & $285 \%$ & 0 & 0 & 0 & $0.00 \%$ \\
\hline Energy & 2 & 0 & 2 & $13.21 \%$ & 0 & 0 & 0 & $0.00 \%$ & 0 & 0 & 0 & $0.00 \%$ \\
\hline Other Finance & 2 & 2 & 4 & $11.12 \%$ & 2 & 0 & 2 & $7.14 \%$ & 1 & 0 & 1 & $3.02 \%$ \\
\hline Total & 117 & 15 & 132 & $3.77 \%$ & 58 & 6 & 64 & $1.79 \%$ & 27 & 4 & 31 & $0.86 \%$ \\
\hline
\end{tabular}

\section{Results}

\subsection{Descriptive Statistics}

Table 2 presents descriptive statistics for the companies that reported SDs (disclosure group) and for those that did not (control group).

The disclosure group was more likely to change CEOs (TO, $\chi^{2}=5.51, \mathrm{p}<0.01$ ), issued more restatements (RESTATEMENT, $\mathrm{t}=2.18, \mathrm{p}=0.04)$, and engaged in more M\&As $\left(\mathrm{M} \& \mathrm{~A}, \chi^{2}=7.71, \mathrm{p}<0.01\right)$ than the control group. The disclosure group also posed a greater risk than the control group $\left(\mathrm{GC}, \chi^{2}=17.44, \mathrm{p}<0.01 ; \mathrm{ROA}, \mathrm{t}=\right.$ 
-17.36; CFO/A, $\mathrm{t}=-10.22, \mathrm{p}<0.01$, GROWTH, $\mathrm{t}=-2.98, \mathrm{p}<0.01$; DEBT, $\mathrm{t}=11.98, \mathrm{p}<0.01)$. The SD disclosure companies are smaller (LNSIZE, $\mathrm{t}=-6.31, \mathrm{p}<0.01$ ), have fewer directors (BOARDSIZE, $\mathrm{t}=-4.87, \mathrm{p}<0.01$ ) with less average service (OFFICE, $\mathrm{t}=-4.69, \mathrm{p}<0.01$ ) and have a higher proportion of outside directors $($ OUTSIDE, $\mathrm{t}=$ $4.15, \mathrm{p}<0.01)($ Note 20).

Table 2. Descriptive statistics of SD disclosure group and control group*

\begin{tabular}{|c|c|c|c|c|c|c|c|}
\hline \multirow[b]{3}{*}{ Variable } & \multicolumn{3}{|c|}{ SD disclosure group } & \multicolumn{3}{|c|}{ Control Group } & \multirow{3}{*}{$\begin{array}{l}\text { Differences } \\
\text { tor }\left(x^{2}\right)\end{array}$} \\
\hline & \multicolumn{3}{|c|}{$(\mathrm{N}=227)$} & \multicolumn{3}{|c|}{$(\mathrm{N}=9,321)$} & \\
\hline & Mean & Median & Std.dev. & Mean & Median & Std.dev. & \\
\hline TO & 0.32 & 0.38 & 0.46 & 0.14 & 0.00 & 0.34 & $5.51^{* * *}$ \\
\hline RESTATEMENT & 1.36 & 1.01 & 2.58 & 0.09 & 0.00 & 0.28 & $2.18^{* *}$ \\
\hline$M \& A$ & 0.12 & 0.00 & 0.32 & 0.07 & 0.00 & 0.25 & $7.71^{* * *}$ \\
\hline$G C$ & 0.25 & 0.22 & 0.42 & 0.03 & 0.00 & 0.16 & $17.44^{* * *}$ \\
\hline$R O A$ & -22.08 & 1.81 & 20.35 & 1.72 & 1.03 & 1.28 & $-17.36^{* * *}$ \\
\hline CFO/A & -2.33 & 1.81 & 19.02 & 6.01 & 7.51 & 10.03 & $-10.22^{* * *}$ \\
\hline GROWTH & -4.59 & -1.21 & 12.04 & 1.36 & 0.78 & 20.53 & $-2.98^{* * *}$ \\
\hline$D E B T$ & 57.61 & 58.36 & 38.67 & 49.18 & 49.91 & 21.09 & $11.98^{* * *}$ \\
\hline LNSIZE & 9.32 & 9.34 & 1.89 & 10.47 & 10.31 & 1.71 & $-6.31^{* * *}$ \\
\hline LNSUB & 1.65 & 1.61 & 1.22 & 1.88 & 1.79 & 1.31 & -1.44 \\
\hline LNSSEGMENTS & 1.29 & 1.22 & 1.17 & 1.67 & 1.69 & 0.74 & $-2.51^{* *}$ \\
\hline FOREIGNSALES & 8.99 & 0.88 & 20.32 & 9.76 & 4.78 & 18.42 & -0.81 \\
\hline$M T B$ & 0.64 & 0.55 & 1.24 & 0.82 & 0.72 & 1.02 & $-5.92^{* * *}$ \\
\hline$R \& D$ & 1.92 & 1.29 & 3.22 & 3.01 & 3.24 & 1.88 & $-2.92^{* *}$ \\
\hline BOARDSIZE & 9.47 & 8.88 & 3.55 & 12.65 & 11.99 & 4.22 & $-4.87 * * *$ \\
\hline OFFICE & 5.04 & 4.52 & 3.02 & 6.51 & 5.54 & 3.44 & $-4.69 * * *$ \\
\hline OUTSIDE & 12.77 & 11.25 & 14.85 & 9.88 & 4.48 & 12.16 & $4.15^{* * *}$ \\
\hline EXPERT & 11.22 & 8.39 & 11.10 & 10.11 & 9.34 & 9.52 & 1.49 \\
\hline DIRECOWN & 11.22 & 10.14 & 14.88 & 9.73 & 10.05 & 14.38 & 1.14 \\
\hline BIGSHARE & 30.88 & 31.55 & 19.56 & 24.77 & 20.99 & 26.88 & $3.02^{* * *}$ \\
\hline FOREIGNOWN & 3.62 & 1.41 & 5.26 & 7.59 & 3.95 & 6.38 & $-3.62^{* * *}$ \\
\hline BANKING & 8.46 & 5.29 & 10.34 & 18.25 & 9.67 & 15.29 & $-7.26^{* * *}$ \\
\hline TRUST & 0.92 & 0.00 & 1.99 & 1.95 & 0.89 & 3.57 & $-2.89^{* *}$ \\
\hline BIG4 & 0.02 & 0.01 & 0.14 & 0.03 & 0.00 & 0.16 & $-25.91^{* * *}$ \\
\hline AUDITORS & 11.39 & 9.22 & 8.63 & 14.22 & 12.01 & 11.74 & $-8.71^{* * *}$ \\
\hline
\end{tabular}

The correlation matrix in Table 3 shows that TO correlates positively with RESTATEMENT, DEBT, LNSIZE, and LNSEGMENTS and negatively with ROA, CFO/A, and GROWTH. Although TO correlates positively with OUTSIDE, it correlates negatively with EXPERT. In terms of ownership, TO correlates negatively with DIRECOWN and positively with BIGSHARE.

Table 3. Correlation matrix (Spearman | Pearson)*

\begin{tabular}{|c|c|c|c|c|c|c|c|c|c|c|c|}
\hline & TO & RESTATEMENT & ROA & CFO/A & GROWTH & DEBT & LNSIZE & LNSUB & LNSEGMENTS & FOREIGNSALE & MTB \\
\hline TO & 1.000 & $0.022^{\circ}$ & $-0.030^{* * *}$ & $-0.003^{* *}$ & $-0.071^{* *}$ & $0.040^{* *}$ & $0.032 * *$ & 0.018 & $0.0377^{* *}$ & 0.010 & $0.026{ }^{* *}$ \\
\hline RESTATEMENT & $0.022^{* *}$ & 1.000 & $-0.046^{* *}$ & $-0.026^{*}$ & $-0.032^{* *}$ & $0.071 * *$ & $-0.088^{* *}$ & 0.004 & 0.017 & $0.020^{* *}$ & 0.015 \\
\hline ROA & $-0.030^{\circ *}$ & $-0.460^{* *}$ & 1.000 & $0.528^{* *}$ & 0.015 & $-0.459 \cdot *$ & $0.143^{* *}$ & 0.010 & $0.024^{*}$ & -0.012 & $0.224^{* *}$ \\
\hline CFO/A & $-0.030^{*}$ & $-0.026^{*}$ & $0.528^{* *}$ & 1.000 & -0.018 & $-0.248^{* *}$ & $0.097 *$ & 0.003 & 0.010 & 0.010 & $0.0311^{* *}$ \\
\hline GROWIH & $-0.070 * *$ & $-0.032^{* *}$ & 0.015 & -0.018 & 1.000 & $0.065 * *$ & $0.274^{* *}$ & $0.197 * *$ & $0.164^{* *}$ & $0.732^{* *}$ & $0.332^{* *}$ \\
\hline DEBT & $0.040^{* *}$ & $0.071 * *$ & $-0.459^{* *}$ & $-0.248^{* *}$ & $0.065 * *$ & 1.000 & $0.110^{* *}$ & $0.148^{* *}$ & $0.159 * *$ & $-0.050 * *$ & $0.091 *$ \\
\hline LNSZZE & $0.032 * *$ & -0.020 & $0.143^{* *}$ & $0.097 * *$ & $0.27 \mathrm{~s}^{\circ *}$ & $0.110^{* * *}$ & 1.000 & $0.698^{* *}$ & $0.632 * *$ & $0.274^{* *}$ & $0.567 * *$ \\
\hline LNSUB & 0.018 & 0.004 & 0.008 & 0.003 & $0.195 *$ & $0.148^{* *}$ & $0.693^{* *}$ & 1.000 & $0.698^{* *}$ & $0.3799^{\circ *}$ & $0.221 *$ \\
\hline LNSEGMENTS & $0.034 * *$ & 0.017 & $0.024^{*}$ & 0.010 & $0.164^{* *}$ & $0.155^{* *}$ & $0.635^{* *}$ & $0.688^{* *}$ & 1.000 & $0.370^{* *}$ & 0.017 \\
\hline FOREIGNSALE & 0.010 & -0.002 & -0.012 & -0.001 & $0.732^{* *}$ & $-0.050^{* * *}$ & $0.274^{* *}$ & $0.381^{* *}$ & $0.372^{* *}$ & 1.000 & $0.192 * *$ \\
\hline MTB & $0.026^{* *}$ & 0.017 & $0.224 * *$ & $0.029 * *$ & $0.332 * *$ & $0.092 * *$ & $0.567 * *$ & $0.222^{*}$ & 0.019 & $0.192 * *$ & 1.000 \\
\hline$R \& D$ & $0.055^{\circ *}$ & 0.005 & 0.018 & 0.011 & $0.224^{* *}$ & 0.015 & $0.422^{* *}$ & $0.331 *$ & $0.223^{* *}$ & $0.155^{* *}$ & $0.168^{\circ *}$ \\
\hline BOARDSZZE & $0.036^{* *}$ & $-0.026^{*}$ & $0.058 * *$ & $0.033^{* *}$ & $0.055^{* *}$ & $0.073^{* *}$ & $0.577^{* *}$ & $0.278^{* *}$ & $0.465^{* *}$ & $0.170^{* *}$ & $0.058 * *$ \\
\hline OFFCE & $-0.146^{* *}$ & -0.012 & $0.049 * *$ & $0.0311^{* *}$ & $-0.198 * *$ & $-0.119 * *$ & $-0.128^{* *}$ & $-0.044^{* *}$ & $-0.164^{* *}$ & $-0.144^{* *}$ & $-0.009 * *$ \\
\hline OUTSIDE & $0.050^{\circ *}$ & 0.017 & $-0.039 * *$ & $-0.025^{*}$ & $0.433^{* *}$ & $0.021^{\circ}$ & 0.003 & 0.002 & $0.0566^{* *}$ & 0.055** & $0.037 * *$ \\
\hline EXPERT & $-0.039 * *$ & 0.001 & 0.014 & 0.016 & $-0.424^{* *}$ & $-0.058 * *$ & $-0.0477^{* *}$ & $-0.077^{* * *}$ & $-0.053 * *$ & $-0.099 * *$ & $-0.047 * *$ \\
\hline DIRECOWN & $-0.105^{* *}$ & $0.023^{*}$ & 0.015 & $0.028^{* *}$ & $-0.128^{* *}$ & $-0.041 *$ & $-0.422^{* *}$ & $-0.588 *$ & $-0.318^{* *}$ & $-0.165^{* *}$ & $-0.223 * *$ \\
\hline BIGSHARE & $0.038^{* *}$ & $0.021^{\circ}$ & $-0.087 * *$ & $-0.062^{* *}$ & $0.1111^{* *}$ & $0.028^{* *}$ & $-0.472^{* *}$ & $-0.269 *$ & $-0.159 * *$ & $-0.255^{* *}$ & -0.007 \\
\hline FOREIGNOWN & 0.015 & -0.004 & $0.260 * *$ & $0.168^{* *}$ & $0.243^{* *}$ & 0.011 & $0.005^{* *}$ & $0.588^{* *}$ & 0.015 & $0.233^{* *}$ & 0.008 \\
\hline BANELNG & 0.010 & $-0.054^{* *}$ & $0.100^{* *}$ & $0.111 * *$ & $0.222^{* *}$ & $0.145^{* *}$ & $0.688^{* *}$ & $0.298^{* *}$ & 0.123 & $0.280^{* *}$ & 0.017 \\
\hline TRUST & 0.008 & 0.006 & $0.332 *$ & $0.234^{* *}$ & $0.517 \%$ & $0.033^{* *}$ & $0.565^{* *}$ & $0.477 *$ & $0.480 * *$ & $0.236^{\circ *}$ & $0.112^{* *}$ \\
\hline
\end{tabular}

Asterisks* and ${ }^{* *}$ indicate two-tailed significance at the $0.05,0.01$ levels, respectively 
Table 3. (Continued)*

\begin{tabular}{|c|c|c|c|c|c|c|c|c|c|c|}
\hline & $R \& D$ & BOARDSIZE & OFFICE & OUTSIDE & EXPERT & DIRECOWN & BIGSHARE & FOREIGNOWN & BANKING & TRUST \\
\hline TO & $0.054^{* *}$ & $0.036 * *$ & $-0.146^{* *}$ & $0.050^{\circ * *}$ & $-0.039 \cdot *$ & $-0.105 * *$ & $0.038^{* *}$ & 0.015 & 0.010 & 0.009 \\
\hline RESTATEMENT & 0.005 & $-0.032 * *$ & -0.012 & $0.068 * *$ & $-0.025^{*}$ & $0.092 * *$ & $0.021^{*}$ & -0.005 & $-0.054^{* *}$ & 0.006 \\
\hline$R O A$ & 0.017 & $0.058^{* *}$ & $0.049 * *$ & $-0.039 \%$ & 0.014 & 0.015 & $-0.087 * *$ & $0.261 *$ & $0.106^{* *}$ & $0.322^{* *}$ \\
\hline CFO/A & 0.011 & $0.033^{* *}$ & $0.031 *$ & $-0.025^{*}$ & 0.016 & $0.028^{*}$ & $-0.062 * *$ & $0.168^{* *}$ & $0.111 * *$ & $0.235 * *$ \\
\hline GRONTH & $0.243^{* *}$ & $0.055 * *$ & $-0.198 * *$ & $0.4333^{* *}$ & $-0.424^{* *}$ & $-0.128^{* *}$ & $0.110^{* *}$ & $0.243 * *$ & $0.221 * *$ & $0.517 * *$ \\
\hline DEBT & 0.009 & $0.073 * *$ & $-0.119 * *$ & $0.021^{*}$ & $-0.058 * *$ & $-0.041 * *$ & $0.028 *$ & 0.012 & $0.146^{* *}$ & $0.034^{* *}$ \\
\hline LNSIZE & $0.422 *$ & $0.575^{* *}$ & $-0.128^{* *}$ & 0.005 & $-0.047 * *$ & $-0.424 * *$ & $-0.472 * *$ & $0.605 *$ & $0.690 * *$ & $0.565^{* *}$ \\
\hline LNSUB & $0.331 *$ & $0.278^{* *}$ & $-0.046 * *$ & 0.005 & $-0.077 * *$ & $-0.553 * *$ & $-0.266^{* *}$ & $0.579 \cdot *$ & $0.298 * *$ & $0.449 * *$ \\
\hline LNSEGMENTS & $0.212^{* *}$ & $0.465 * *$ & $-0.164 * *$ & $0.056 * *$ & $-0.053 * *$ & $-0.319 * *$ & $-0.159 * *$ & 0.017 & $0.123 * *$ & $0.482 * *$ \\
\hline FOREIGNSALE & $0.155^{* *}$ & $0.172^{* *}$ & $-0.164^{* *}$ & $0.056^{\circ * *}$ & $-0.053^{* *}$ & $-0.166^{* *}$ & $-0.256^{* * *}$ & $0.233^{* *}$ & $0.281^{* *}$ & $0.236^{* *}$ \\
\hline MTB & $0.169 * *$ & $0.055^{* *}$ & -0.007 & $0.034^{* *}$ & $-0.046 * *$ & $-0.022^{* *}$ & -0.003 & 0.008 & 0.015 & $0.124^{* *}$ \\
\hline$R \& D$ & 1.000 & 0.009 & 0.007 & $0.142^{* *}$ & 0.007 & $-0.058^{* *}$ & -0.011 & $0.339 * *$ & 0.003 & $0.124 * *$ \\
\hline BOARDSIZE & 0.009 & 1.000 & $-0.141^{* *}$ & $0.030^{\circ * *}$ & $-0.085^{* *}$ & $-0.276^{* *}$ & $-0.281 *$ & $0.358^{* *}$ & $0.508^{* *}$ & $0.365^{* *}$ \\
\hline OFFICE & 0.007 & $-0.141 * *$ & 1.000 & $-0.224^{* *}$ & $0.156^{* *}$ & $0.301 * *$ & $-0.121 * *$ & $-0.1133^{* *}$ & -0.004 & -0.009 \\
\hline OUTSIDE & $0.144 * *$ & $0.301 * *$ & $-0.224 * *$ & 1.000 & 0.001 & $-0.068 * *$ & $0.223^{* *}$ & $-0.087 \%$ & $-0.344^{* *}$ & $-0.171 * *$ \\
\hline EXPERT & 0.007 & $-0.085 * *$ & $0.156^{* *}$ & 0.001 & 1.000 & $0.151 * *$ & $-0.022^{*}$ & 0.002 & -0.015 & -0.011 \\
\hline DIRECOWN & $-0.058 * *$ & $-0.276^{* *}$ & $0.300 \%$ & $-0.068 *$ & $0.152^{* *}$ & 1.000 & $0.046^{* *}$ & $-0.322^{* *}$ & $-0.370^{\circ *}$ & $-0.243^{* *}$ \\
\hline BIGSHARE & -0.011 & $-0.280 * *$ & $-0.121 * *$ & $0.222 * *$ & $0.002^{*}$ & $0.046 * *$ & 1.000 & $-0.239 * *$ & $-0.651 * *$ & $-0.050^{* * *}$ \\
\hline FOREIGNOWN & $0.443 *$ & $0.359 * *$ & $-0.112 *$ & $-0.088 * *$ & 0.002 & $-0.322^{* *}$ & $-0.501 *$ & 1.000 & $0.228^{*}$ & $0.260^{* *}$ \\
\hline BANKLNG & 0.003 & $0.508 * *$ & -0.003 & $-0.345^{\circ * *}$ & -0.015 & $-0.370^{*}$ & $-0.651^{* * *}$ & $0.228 *$ & 1.000 & $-0.050 * *$ \\
\hline TRUST & $0.122^{* *}$ & $0.355 * *$ & -0.009 & $-0.171 *$ & -0.012 & $-0.243^{* *}$ & $-0.050^{\circ * *}$ & $0.269 * *$ & $-0.050=$ & 1.000 \\
\hline
\end{tabular}

Asterisks* and ${ }^{* *}$ indicate two-tailed significance at the $0.05,0.01$ levels, respectively.

\subsection{Regression Analysis}

\subsubsection{Disclosure of Significant Deficiencies and Replacing the CEO}

Table 4 (Panel A) shows the results of the binary probit regression for Model (1). The results indicate a statistically significant positive correlation between SD and TO $(z=3.772, p<0.001)$, which supports Hypothesis 1 and suggests that a SD disclosure in the ICFR is a factor in replacing the CEO. Among the variables related to corporate governance, EXPERT $(\mathrm{z}=-2.135, \mathrm{p}=0.043)$ correlates negatively with TO, whereas OUTSIDE has no relation with TO. Table 4 (Panels B and C) shows the results of the probit regression for Model (2). Although SD correlates significantly and positively with OUTSIDE $(\mathrm{z}=2.552, \mathrm{p}<0.037)$, it has no correlation with EXPERT (Note 21$)$.

Table 4. Probit Regression

Panel A: Model (1) DV=TO; Panel B: Model (2) DV=OUTSIDE; Panel C: Model (2) DV=EXPERT

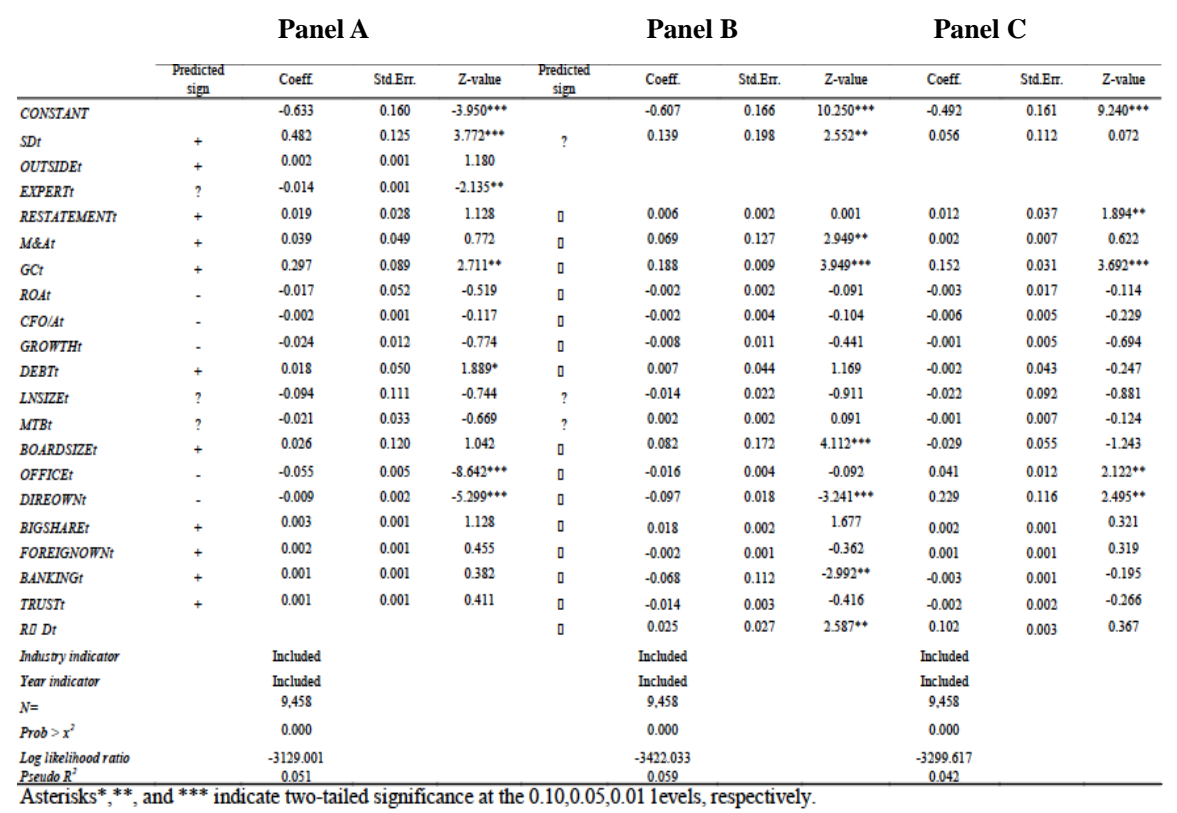




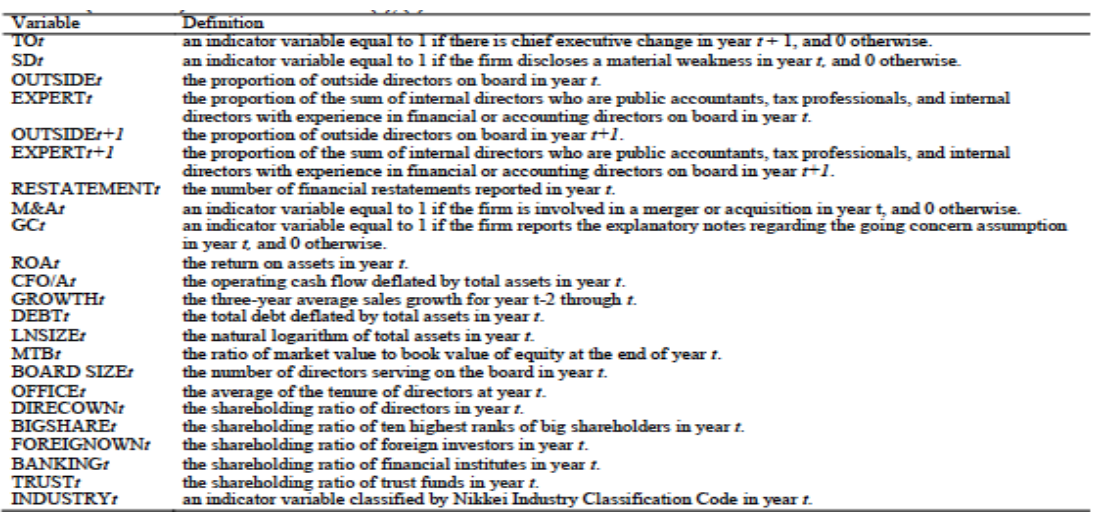

\section{Remediation of Significant Deficiencies}

The next question arising from these results is whether replacing the CEO and upgrading the independence and expertise of corporate governance prompts SD remediation. Table 5 (Panel A) shows the results of the logistic regression for Model (3). The results suggest that TO is not significantly correlated with REMEDIATION (Wald = $2.294, \mathrm{p}=0.141$ ). Although a change in the board's independence correlates negatively with REMEDIATION (Wald $=3.975, \mathrm{p}=0,046)$, a change in the board's expertise $(\triangle \mathrm{EXPERT})$ correlates positively (Wald $=7.451, \mathrm{p}=0.007)$, which suggests that corporations with expert board members are more likely to remediate SDs in the short term. However, even if a corporation increases the independence of the board after SD disclosure, the change does not induce remediation by the next fiscal year end. Instead, firms enhancing board independence are less likely to remediate SDs; therefore, this action appears to camouflage the situation, which makes it difficult to exclude the CEO's influence.

Table 5 (Panel B) presents the relationship between REMEDIATION and TO following enhancements to the board's independence and expertise (Model 4). The interactions between TO and $\triangle$ OUTSIDE and TO and $\triangle$ EXPERT are not significantly correlated with REMEDIATION, which indicates that replacing the CEO does not affect remediation regardless of whether a firm reforms its corporate governance.

Table 5. Logistic Regression

\begin{tabular}{|c|c|c|c|c|c|}
\hline & \multirow[b]{2}{*}{$\begin{array}{c}\text { Predicted } \\
\text { sign }\end{array}$} & \multicolumn{2}{|c|}{$\begin{array}{c}\text { Panel A: Model (3) } \\
\text { Pane1 A: DV=REMEDIATE }\end{array}$} & \multicolumn{2}{|c|}{$\begin{array}{c}\text { Pane1 B: Model (4) } \\
\text { Pane1 B: DV=REMEDIATE }\end{array}$} \\
\hline & & Coeff. & Wald & Coeff & Wald \\
\hline CONSTANT & & 4.523 & $6.880 * *$ & 3.310 & $7.705 * *$ \\
\hline TO & $+/-$ & -2.703 & 2.276 & -2.057 & 2.009 \\
\hline TO* $\triangle O U T S I D E$ & + & & & -0.073 & 0.211 \\
\hline TO $\triangle E X P E R T$ & + & & & 0.451 & 0.619 \\
\hline ENVIRONMW & - & -2.477 & $4.882 * *$ & -2.025 & $5.011 * *$ \\
\hline$\triangle L N S I Z E$ & + & 0.606 & 0.242 & 0.566 & 0.120 \\
\hline$\triangle L N S E G M E N T S$ & - & -0.373 & 0.438 & -2.158 & 0.225 \\
\hline$\triangle F O R E I G N S A L E$ & - & -0.116 & 1.446 & -0.082 & 1.157 \\
\hline$M \& A$ & - & 4.730 & 2.050 & 2.879 & 1.928 \\
\hline$\triangle R O A$ & + & 0.143 & $2.989=$ & 0.166 & $3.056^{*}$ \\
\hline$\triangle C F O / A$ & + & 0.247 & $2.985^{*}$ & 0.157 & $2.849 *$ \\
\hline$G C$ & - & -2.610 & 1.549 & -3.754 & $3.575 *$ \\
\hline BIG4 & + & 5.893 & $3.293=$ & 2.927 & 1.740 \\
\hline$\triangle A U D N U M B E R$ & + & 0.578 & 2.111 & 0.611 & 0.123 \\
\hline$\triangle O U T S I D E$ & + & -0.179 & $3.724 * *$ & -0.109 & $2.849 *$ \\
\hline$\triangle E X P E R T$ & + & 0.728 & $7.694 * *$ & 0.655 & $4.961 * *$ \\
\hline$\triangle D I R E C O W N$ & - & -0.038 & 0.294 & -0.074 & 0.544 \\
\hline$\triangle B I G S H A R E$ & + & -0.369 & 2.063 & -0.128 & 2.553 \\
\hline$\triangle F O R E I G N O W N$ & + & 0.024 & 0.036 & 0.019 & 0.024 \\
\hline$\triangle B A N K I N G$ & + & -1.004 & $3.351 *$ & -0.992 & 1.675 \\
\hline$\triangle T R U S T$ & + & 0.147 & 0.172 & 0.059 & 0.116 \\
\hline Industry indicator & & Included & & Included & \\
\hline$N=$ & & 165 & & 165 & \\
\hline Remediation $N=$ & & 127 & & 127 & \\
\hline Prob $>x^{2}$ & & 0.000 & & 0.000 & \\
\hline -2 Log likelihood ratio & & 28.984 & & 19.508 & \\
\hline Pseudo $R^{2}$ & & 0.347 & & 0.402 & \\
\hline
\end{tabular}




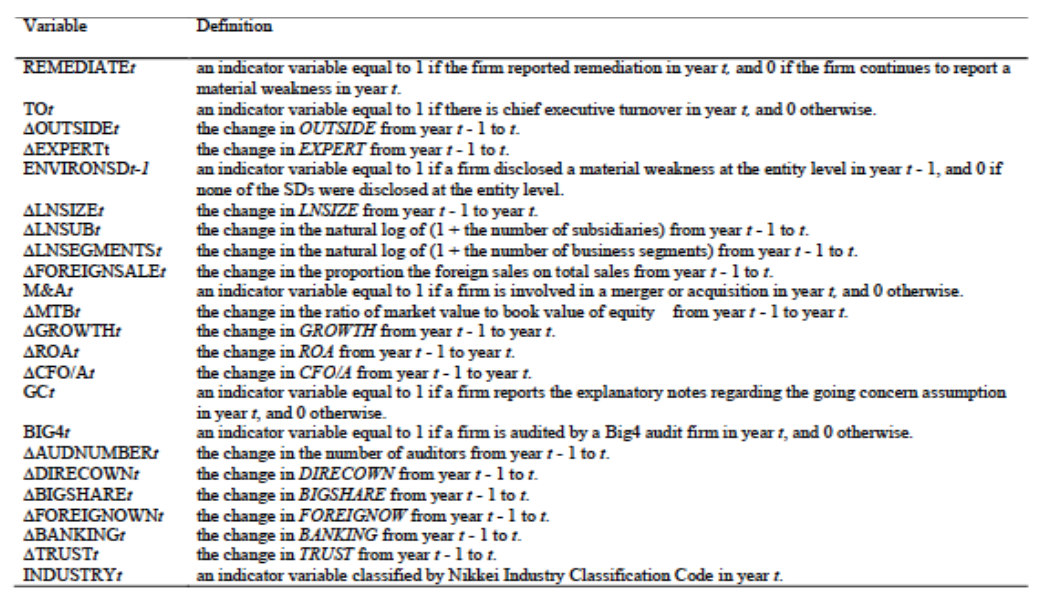

\subsection{Additional Analysis}

Replacing the CEO and changes in audit fees. Munsif, Raghunandan, Rama, \& Singhvi (2011) show that firms that remediate SDs have lower audit fees than firms that continue to report them, and Hoag \& Hollingworth (2011) show that audit fees decline for companies that remediate SDs. Feldmann, Read, \& Abdolmohammadi (2009) find that replacing the CFO moderates subsequent increases in audit fees for corporations that modified and restated their financials (Note 22). Several empirical studies link fees with auditors' perceptions of clients' control risks (Hay, Knechel, \& Wong, 2006). In short, previous research suggests that the disclosure of a SD increases audit fees (Note 23) and that auditors moderate fee increases if they believe that replacing the CEO and board members reduces control risks (Feldmann, Read, \& Abdolmohammadi, 2009). However, this study's results suggest that replacing the CEO does not inherently remediate SDs by improving internal controls. The question then arises as to whether auditors perceive that replacing the CEO in itself reduces the control risk as reflected in auditing fees, which is tested using Model (5): (Note 24)

CHANGEFEEit $=\beta 0+\beta 1$ TOit $+\beta 2$ REMEDIATEit $+\beta 3 \Delta$ OUTSIDEit $+\beta 4 \Delta$ EXPERTit $+\beta 5 \Delta$ RESTATEMENTit

$+\beta 6 \Delta$ LNSIZEit $+\beta 7 \Delta$ M\&Ait $+\beta 8 \Delta$ GROWTHit $+\beta 9 \Delta$ LNSEGMENTSit $+\beta 10 \Delta$ FOREIGNSALEit

$+\beta 11 \Delta$ INVENTORYit $+\beta 12$ GCit $+\beta 13 \Delta$ ROAit $+\beta 14 \Delta$ DEBTit $+\beta 15$ BIG 4 it

$+\beta 16 \triangle$ AUDNUMBERit $+\beta 17$ INDUSTRYit $+\varepsilon$.

CHANGEFEEit $=\beta 0+\beta 1$ TOit $+\beta 2$ REMEDIATEit $+\beta 3$ REMEDIATEit $*$ TOit $+\beta 4$ REMEDIATERTit*OUTSIDEit

$+\beta 5$ REMEDIATERTit*EXPERTit $+\beta 6 \Delta$ OUTSIDEit $+\beta 7 \Delta$ EXPERTit

$+\beta 8 \Delta$ RESTATEMENTSit $+\beta 9 \Delta$ LNSIZEit $+\beta 10$ M \&Ait $+\beta 11 \Delta$ GROWTHit

$+\beta 12 \Delta$ LNSEGMENTit $+\beta 13 \Delta$ FOREIGNSALEit $+\beta 14 \Delta$ INVENTORYit

$+\beta 15 \Delta$ ROAit $+\beta 16 \Delta$ DEBTit $+\beta 17$ BIG4it $+\beta 18 \Delta$ AUDNUMBERit+ $+\beta 19$ INDUSTRYit $+\varepsilon$.

Table 6 describes the results of the ordinary least squares regression for Models (5) and (6) (Note 25). The results suggest that REMEDIATE correlates negatively with CHANGEFEE (Wald $=-2.285, p=0.025$ ). The variables TO, $\triangle$ OUTSIDE, and $\triangle$ EXPERT are not significantly correlated with CHANGEFEE. However, interactions between REMEDIATION and $\triangle$ EXPERT correlate significantly and negatively with changes in audit fees (Wald $=-1.750, p$ $=0.084)$. These results show that remediating SDs affects auditors' assessment of the control risk and audit fees only when remediation is coupled with a change in corporate governance expertise. 
Table 6. OLS Regression

\begin{tabular}{|c|c|c|c|c|c|}
\hline & \multirow[b]{2}{*}{ Predicted sizm } & \multicolumn{2}{|c|}{$\begin{array}{c}\text { Model (5) } \\
\text { Panel A:DV }=\text { CHANGEFEE }\end{array}$} & \multicolumn{2}{|c|}{$\begin{array}{c}\text { Model (6) } \\
\text { PanelB: DV }=\text { CHANGEFEE }\end{array}$} \\
\hline & & Coeff. & trvalue & Coeff. & Wald \\
\hline CONSTANT & & 0.379 & $2.444^{* *}$ & 0.795 & $2.583^{* *}$ \\
\hline TO & - & 0.062 & 0.593 & 0.060 & 0.557 \\
\hline REMEDLATE & - & -0.245 & $-2.285^{* *}$ & -0.532 & $-2.551^{* *}$ \\
\hline REME*ATO & - & & & 0.173 & 1.617 \\
\hline REME* $\triangle O U T S I D E$ & - & & & 0.108 & 0.928 \\
\hline$R E M E * \triangle E X P E R T$ & - & & & -0.227 & $-1.750^{\circ}$ \\
\hline$\triangle O U T S I D E$ & - & 0.032 & 0.276 & 0.053 & 0.527 \\
\hline$\triangle E X P E R T$ & - & -0.214 & -0.449 & -0.243 & -0.229 \\
\hline$\triangle R E S T A T E M E N T$ & + & -0.011 & -0.119 & -0.009 & -0.194 \\
\hline ALNSIZE & + & 0.291 & $2.914^{* *}$ & 0.314 & $3.035^{* *}$ \\
\hline M\&A & + & 0.037 & 0.707 & 0.054 & 0.554 \\
\hline$\triangle G R O W T H$ & + & -0.085 & -0.911 & -0.080 & -0.738 \\
\hline$\triangle L N S E G M E N T$ & + & 0.143 & 1.121 & 0.129 & 1.442 \\
\hline$\triangle F O R E I G N S A L E$ & + & 0.199 & $1.928^{*}$ & 0.180 & $1.902^{*}$ \\
\hline$\triangle D N V E N T O R Y$ & + & 0.025 & 0.721 & 0.045 & 0.423 \\
\hline$G C$ & + & 0.051 & 0.462 & 0.057 & 0.518 \\
\hline$\triangle R O A$ & - & -0.117 & -1.539 & -0.159 & -1.581 \\
\hline$\triangle D E B T$ & + & 0.179 & $1.882^{*}$ & 0.152 & 1.268 \\
\hline BIG4 & + & 0.168 & $1.926^{*}$ & 0.164 & 1.595 \\
\hline$\triangle A U D N U M B E R$ & + & 0.198 & $2.081^{* *}$ & 0.185 & $1.933^{*}$ \\
\hline Industry indicator & & Included & & Inchuded & \\
\hline$N=$ & & 165 & & 165 & \\
\hline Romediation $N=$ & & 127 & & 127 & \\
\hline Adiustad- $R^{2}$ & & 0.144 & & 0.148 & \\
\hline F-vaine & & $2143 / n=001$ & & $1004 / n=01$ & \\
\hline Variable & Definition & & & & \\
\hline CHNAGEFEE $t$ & the change in as & 5 from year $t$ - & & & \\
\hline TOt & an indicator vari & gual to 1 if the & cutive tums & and 0 oth & \\
\hline REMEDIATE $t$ & $\begin{array}{l}\text { an indicator vari } \\
\text { a material weakr }\end{array}$ & $\begin{array}{l}\text { gual } 1 \text { if a fim } \\
\text { year } t \text {. }\end{array}$ & ediation in & if a firm co & Ited \\
\hline$\Delta O U T S I D E t$ & the change in $O$ & $E$ from year $t$ & & & \\
\hline$\triangle E X P E R T t$ & the change in $E$. & from year $t$ - & & & \\
\hline$\triangle R E S T A T E M E N T t$ & the change in the & er of financia & revorted fir & to $t$. & \\
\hline$\triangle L N S I Z E t$ & the change in $L$. & from vear $t-1$ & & & \\
\hline M\&At & an indicator vari & qual to 1 if a fi. & In a merge & ion in year & \\
\hline$\Delta \mathrm{GROWTHt}$ & the change in GI & $H$ from vear $t$ & & & \\
\hline$\triangle L N S E G M E N T S t$ & the change in the & al $\log$ of $(1+$ & business se & 2 year $t-1$ & \\
\hline$\triangle$ FOREIGNSALE $t$ & the change in the & ortion of forei & 1 sales fror & & \\
\hline$\triangle D N V E N T R Y t$ & the change in the & atorv / total as & $t-1$ to $t$ & & \\
\hline & an indicator van & coual to 1 if a & he explans & garding th & Assumbtion in \\
\hline$\triangle R O A t$ & the change in $R C$ & m year $t-1$ to & & & \\
\hline$\triangle \mathrm{DEBT} t$ & the change in $D$ & om year $t-1 \mathrm{t}$ & & & \\
\hline BIGAt & an indicator vari & gual to 1 if a $f$ & oy a Bigt a & rear $t$, and & \\
\hline$\triangle A U D N U M B E R t$ & the change in the & per of auditors & to $t$ & & \\
\hline MDUSTRY & an indicator vari & assified he $\mathrm{N}$ & Classificati & eart & \\
\hline
\end{tabular}

\section{Conclusions}

This study examines Japanese corporations that disclose SDs in ICFR and determines whether replacing the CEO and enhancing board members' independence and financial expertise are followed by SD remediation. The results indicate that Japanese companies that disclose SDs in ICFR are more likely to replace their CEOs and enhance board independence. In addition, the results indicate that although these actions do not affect SD remediation, upgrading the board's accounting expertise correlates positively with SD remediation. The results have not consistently paralleled results that focus on firms in the U.S.

Until recently, regulators (e.g., Japanese Legislative Council of the Ministry of Justice, Tokyo Stock Exchange) addressed issues of fraud and substandard financial controls by attempting to enhance the independence of corporate boards (Note 26). However, the results indicate that the board members' expertise and not their independence is the central factor in improving the quality of internal controls. These findings should interest Japan's regulators, auditing standard setters, and users of financial statements when they consider improvements in the quality of internal controls. In particular, these individuals must realize that the control environment is not easily improved in Japanese firms, particularly because new CEOs encounter difficulties in changing the environment established by their predecessors. Furthermore, these individuals should understand the reason current Japanese law emphasizes independence in corporate governance, but they must also evaluate the expertise of internal directors.

Prior studies argue that national culture influences the shaping of corporate governance (Note 27). The distinctive characteristics of organizational management as adopted by most Japanese firms adopt are bottom-up and group-oriented problem solving, and it is important for Japanese firms to create a corporate culture that promotes 
group values and cooperation (Note 28). However, in U.S. firms, almost no attention is focused on group harmony (Note 29). The influences of the differences in national culture and organizational management on shaping corporate governance are by no means small. Naturally, institutions for corporate governance reform are focused on the independence of corporate governance around the world, which is commonly understood. However, if corporate governance reforms are carried out in defiance of any national culture, the change may fail. The effectiveness of corporate governance monitoring should be considered from various perspectives.

\section{Acknowledgements}

I appreciate constructive insights on an earlier version of this paper from participants at the 2011 Annual Conference on Asian Academic Accounting Association, the 2012 Annual congress on European Accounting Association, and the 2012 American Accounting Association Annual Meeting. All errors are my alone.

\section{References}

Abe, N., \& Oguro, Y. (2004). A Semiparametoric analysis of top executive turnover and outside directors. Economic Review, 55(1), 72-84.

Agrawal, A., \& Chadha, S. (2005). Corporate governance and accounting scandals. Journal of Law and Economics, 48(2), 371-406. https://doi.org/10.1086/430808

Agrawal, A., \& Cooper, T. (2014). Insider Trading before accounting scandals. Working paper. Tuscaloosa, AL: University of Alabama.

Ahmadjian, C. L., \& Robinson, P. (2001). Safety in Numbers: Downsizing and the Deinstitutionalization of Permanent Employment in Japan. Administrative Science Quarterly, 46(4), 622-654. https://doi.org/10.2307/3094826

Arthaud-Day, M. L., Certo, S. T., Dalton, C. M. D., \& Dalton, D. R. (2006). A changing of the guard: Executive and director turnover following corporate financial restatements. Academy of Management Journal, 49(6), 1119-1136. https://doi.org/10.5465/amj.2006.23478165

Ashbaugh-Skaife, H., Collins, D. W., Kinney Jr, W. R., \& Lafond, R. (2009). The effect of SOX internal control deficiencies on firm risk and cost of equity. Journal of Accounting Research, 47(1), 1-43. https://doi.org/10.1111/j.1475-679X.2008.00315.x

Bedard, J. C., Hoitash, R., Hoitash, U., \& Westermann, K. (2012). Material weakness remediation and earnings quality: A detailed examination by type of control deficiency. Auditing: A Journal of Practice \& Theory, 31(1), 57-78. https://doi.org/10.2308/ajpt-10190

Beneish, M. D., Billings, M. B., \& Hodder, L. D. (2008). Internal control weaknesses and information uncertainty. The Accounting Review, 83(3), 665-703. https://doi.org/10.2308/accr.2008.83.3.665

Boone, A. L., Casares Field, L., Karpoff, J. M., \& Raheja, C. G. (2007). The determinants of corporate board size and composition: An empirical analysis. Journal of Financial Economics, 85(1), 66-101. https://doi.org/10.1016/j.jfineco.2006.05.004

Burt, I. (2016). An understanding of the differences between internal and external auditors in obtaining information about internal control weaknesses. Journal of Management Accounting Research, 28(3), 83-99. https://doi.org/10.2308/jmar-51471

Carcello, J. V., Neal, T. L., Palmrose, Z. V., \& Scholz, S. (2011). CEO involvement in Selecting Board Members, Audit Committee Effectiveness, and Restatements. Contemporary Accounting Research, 28(2), 396-430. https://doi.org/10.1111/j.1911-3846.2010.01052.x

Choi, J. H., Choi, S., Hogan, C., \& Lee, J. (2013). The effect of human resource investment in internal control on the disclosure of internal control weaknesses. Auditing: A Journal of Practice \& Theory, 32(4), 169-199. https://doi.org/10.2308/ajpt-50514

Chou, L. T. L., \& Wang, Y. F. (2010). Effects of post-SOX restatement characteristics and management behaviour on CEO/CFO turnover. Working paper. National Chengchi University, Providence University.

Coates, J., \& Srinivasan, S. (2014). SOX after ten years: A mmultidisciplinary reveiew. Accounting Horizons, 28(3), 627-671. https://doi.org/10.2308/acch-50759 
Cohen, J. R., Krishnamoorthy, G., \& Wright, A. M. (2008). Form versus substance: The implications for auditing practice and Research of Alternative Perspectives on Corporate Governance. Auditing: A Journal of Practice \& Theory, 27(2), 181-198. https://doi.org/10.2308/aud.2008.27.2.181

Costello, A. M., \& Wittenberg-Moerman, R. (2011). The impact of financial reporting quality on debt contracting: Evidence from internal control weakness reports. Journal of Accounting Research, 49(1), 97-136. https://doi.org/10.1111/j.1475-679X.2010.00388.x

De Franco, G., Guan, Y., \& Lu, H. (2005). The wealth change and redistribution effects of Sarbanes-Oxley internal control disclosures. Working paper. Toronto, Canada: University of Toronto.

Desai, H., Hogan, C. E., \& Wilkins, M. S. (2006). The reputational penalty for aggressive accounting: Earnings restatements and management turnover. The Accounting Review, 81(1), 83-112. https://doi.org/10.2308/accr.2006.81.1.83

Dhaliwal, D., Hogan, C., Trezevant, R., \& Wilkins, M. (2011). Internal control disclosures, monitoring, and the cost of debt. The Accounting Review, 86(4), 1131-1156. https://doi.org/10.2308/accr-10043

Dong, Y., Girardone, C., \& Kuo, J. M. (2016). Governance efficiency and risk taking in Chinese banking. British Accounting Review, 49(2), 211-229. https://doi.org/10.1016/j.bar.2016.08.001

Fama, E. F. (1980). Agency problems and the theory of the firm. Journal of Political Economy, 88(2), 288-307. https://doi.org/10.1086/260866

Feldmann, D. A., Read, W. J., \& Abdolmohammadi, M. J. (2009). Financial restatements, audit fees, and the moderating effect of CFO turnover. Auditing: A Journal of Practice and Theory, 28(1), 205-223. https://doi.org/10.2308/aud.2009.28.1.205

Ge, W., \& McVay, S. (2005). The disclosure of material weaknesses in internal control after the Sarbanes-Oxley Act. Accounting Horizons, 19(3), 137-158. https://doi.org/10.2308/acch.2005.19.3.137

Geiger, M. A., \& Taylor, P. L. (2003). CEO and CFO certifications of financial information. Accounting Horizons, 17(4), 357-368. https://doi.org/10.2308/acch.2003.17.4.357

Ghoshray, S. (2004). Impact of Sarbanes-Oxley on the multiple listed corporations: Conflicts in comparative corporate laws and possible remedies. Journal of International \& comparative Law, 10, 447-457.

Goh, B. W. (2009). Audit committees, Boards of Directors, and Remediation of Material Weaknesses in internal control. Contemporary Accounting Research, 26(2), 549-579. https://doi.org/10.1506/car.26.2.9

Griffin, D. W., Guedhami, O., Kwok, C. C., Li, K., \& Shao, L. (2017). National culture and the value implication of corporate governance. Retrieved from http://www.ssm.com/abstract=2400078.

Hammersley, J. S., Myers, L. A., \& Shakespeare, C. (2008). Market reactions to the disclosure of internal control weaknesses and to the characteristics of those weaknesses under Section 302 of the Sarbanes Oxley act of 2002. Review of Accounting Studies, 13(1), 141-165. https://doi.org/10.1007/s11142-007-9046-z

Hammersley, J. S., Myers, L. A., \& Zhou, J. (2012). The failure to remediate previously disclosed material weaknesses in internal controls. Auditing: A Journal of Practice \& Theory, 31(2), 73-111. https://doi.org/10.2308/ajpt-10268

Hansmann, H., \& Kraakman, R. H. (2001). The end of history for corporate law. Georgetown Law Journal, 89(2), 439-479.

Hatvany, N., \& Pucik, V. (1981). Academy of Management Review, 6(3), 469-480.

Hay, D. C., Knechel, W. R., \& Wong, N. (2006). Audit fees: A meta-analysis of the effect of Supply and demand attributes. Contemporary Accounting Research, 23(1), 141-191. https://doi.org/10.1506/4XR4-KT5V-E8CN-91GX

Hennes, K. M., Leone, A. J., \& Miller, B. P. (2008). The importance of distinguishing errors from irregularities in Restatement Research: The case of restatements and CEO/CFO turnover. The Accounting Review, 83(6), 1487-1519. https://doi.org/10.2308/accr.2008.83.6.1487

Hoag, M. L., \& Hollingsworth, C. W. (2011). An intertemporal analysis of audit fees and Section 404 material weaknesses. Auditing: A Journal of Practice and Theory, 30(2), 173-200. https://doi.org/10.2308/ajpt-50005 
Hogan, C. E., \& Wilkins, M. S. (2008). Evidence on the audit Risk Model: Do Auditors Increase Audit Fees in the presence of internal control deficiencies? Contemporary Accounting Research, 25(1), 219-242. https://doi.org/10.1506/car.25.1.9

Hoitash, R., Hoitash, U., \& Bedard, J. C. (2008). Internal control quality and audit pricing under the Sarbanes-Oxley act. Auditing, 27(1), 105-126. https://doi.org/10.2308/aud.2008.27.1.105

Hoitash, U., Hoitash, R., \& Bedard, J. C. (2009). Corporate governance and internal control over financial reporting: A Comparison of Regulatory Regimes. The Accounting Review, 84(3), 839-867. https://doi.org/10.2308/accr.2009.84.3.839

Hopt, K., \& Leyens, P. C. (2004). Board models in Europe - recent developments of internal corporatate governence structures in Germany, the United Kingdom, France, and Italy. European Corporate Governance Institute (ECGI) - Law Research Paper Series. Working Paprer, No.18.

Johnstone, K. M., Li, C., \& Rupley, K. H. (2011). Changes in corporate governance associated with the Revelation of internal control material weaknesses and their subsequent remediation. Contemporary Accounting Research, 28(1), 331-383. https://doi.org/10.1111/j.1911-3846.2010.01037.x

Kang, J. K., \& Shivdasani, A. (1995). Firm performance, corporate governance, and top executive turnover in Japan. Journal of Financial Economics, 38(1), 29-58. https://doi.org/10.1016/0304-405X(94)00807-D

Kang, J. K., \& Shivdasani, A. (1996). Does the Japanese governance system enhance shareholder wealth? Evidence from the stock-price effects of top management turnover. The Review of Financial Studies, 9(4), 1061-1095. https://doi.org/10.1093/rfs/9.4.1061

Kaplan, S. N. (1994). Top executive rewards and firm Performance: A comparison of Japan and the United States. Journal of Political Economy, 102(3), 510-546. https://doi.org/10.1086/261944

Kawamoto, S. (2009). Internally prompted executives in the twentieth century Japan: General description and factors for their appointment. WIAS Discussion Paper, No. 2009-002. Shinjuku, Japan: Waseda Institute for Advanced Study.

Kim, J. B., Song, B. Y., \& Zhang, L. (2011). Internal control weakness and bank Loan contracting: Evidence from SOX Section 404 disclosures. The Accounting Review, 86(4), 1157-1188. https://doi.org/10.2308/accr-10036

Krishnan, J. (2005). Audit committee quality and internal control: An empirical analysis. The Accounting Review, 80(2), 649-675. https://doi.org/10.2308/accr.2005.80.2.649

Krishnan, J., Rama, D., \& Zhang, Y. (2008). Costs to comply with SOX section 404. Auditing: A Journal of Practice and Theory, 27(1), 169-186. https://doi.org/10.2308/aud.2008.27.1.169

Linck, J. S., Netter, J. M., \& Yang, T. (2008). The determinants of board structure. Journal of Financial Economics, 87(2), 308-328. https://doi.org/10.1016/j.jfineco.2007.03.004

Marden, R. E., Edwards, R. K., \& Stout, W. D. (2003). The CEO/CFO certification requirement. CPA Journal, 73(7), 36-44.

Masulis, R. W., \& Mobbs, S. (2011). Are all inside directors the same? Evidence from the external directorship market. Journal of Finance, 66(3), 823-872. https://doi.org/10.1111/j.1540-6261.2011.01653.x

Menon, K., \& Williams, D. D. (2008). Management turnover following auditor resignations. Contemporary Accounting Research, 25(2), 567-604. https://doi.org/10.1506/car.25.2.10

Miyajima, H., \& Ogawa, R. (2012). Understanding change in board composition: Determinants of board composition and effects of outside directors. RIETI Policy Discussion Paper Series, 12(13). Research Institute of Economy, Trade and Industry.

Mobbs, S. (2013). CEOs under fire: The effects of competition from inside directors on forced CEO turnover and CEO compensation. Journal of Financial and Quantitative Analysis, 48(3), 669-698. https://doi.org/10.1017/S0022109013000318

Mroczkowski, T., \& Hanaoka, M. (1989). Continuity and change in Japanese management. California Management Review, 31(2), 39-53. https://doi.org/10.2307/41166558

Munsif, V., Raghunandan, K., Rama, D. V., \& Singhvi, M. (2011). Audit fees after remediation of internal control weaknesses. Accounting Horizons, 25(1), 87-105. https://doi.org/10.2308/acch.2011.25.1.87 
Ogneva, M., Subramanyam, K. R., \& Raghunandan, K. (2007). Internal control weakness and cost of equity: Evidence from SOX Section 404 disclosures. The Accounting Review, 82(5), 1255-1297. https://doi.org/10.2308/accr.2007.82.5.1255

Ohsawa, T. (2010). Introduction to characteristics of Japanese management style: For better understanding of cultural differences in comparing MBTI data of Japan with that of other countries. Retrieved from www.typeandculture.org/Pages/C_papers93/18Ohsawa.pdf

Ouchi, W. (1981). Theory Z: How American business can meet the Japanese Challenge. Business Horizons, 24(6), 82-83. https://doi.org/10.1016/0007-6813(81)90031-8

Public Company Accounting Oversight Board (PCAOB). (2007). Auditing standard no. 5 - An audit of internal control over financial reporting that is integrated with an audit of financial statements. Washington, DC: PCAOB.

Public Company Accounting Oversight Board (PCAOB). (2008). Audit Considerations in the Current Economic Environment. Staff Audit Practice Alert No.3. Washington, DC: PCAOB.

Raghunandan, K., \& Rama, D. V. (2006). SOX Section 404 material weakness disclosures and audit fees. Auditing: A Journal of Practice and Theory, 25(1), 99-114. https://doi.org/10.2308/aud.2006.25.1.99

Sagi, S. (2015). "Ringi Syatem" the decision making process in japanese management systems: An overview. International Journal of Management and Humanities, 1(7), 10-11.

Sheard, P. (1994). Main banks and the governance of financial distress. In M. Aoki, \& H. Patrick (Eds.), The Japanese Main Bank System (pp. 188-230). Oxford, UK: Oxford University Press.

Simunic, D. A. (1980). The pricing of audit services: Theory and evidence. Journal of Accounting Research, 18(1), 161-190. https://doi.org/10.2307/2490397

Simunic, D. A., \& Stein, M. (1996). Impact of litigation risk on audit pricing: A review of the economics and the evidence. Auditing: A Journal of Practice and Theory, 15(1), 119-134.

Tanikawa, T. (2016). A study of Japanese-style management: Focusing on internal promotion system. Rikkyo Bulletin Business, 12, 59-71.

Tokyo Stock Exchange. (2013). White Paper on Corporate Governance. Tokyo, Japan: TSE.

Williamson, O. E. (2000). The new institutional economics: Taking stock, looking ahead. Journal of Economics Literature, 38(3), 595-613. https://doi.org/10.1257/jel.38.3.595

Yooyanyong, P., \& Muenjohn, N. (2010). Leadership styles of expatriate managers: A comparison between American and Japanese expatriates. Journal of American Academy of Business, 15(2), 161-167.

\section{Notes}

Note 1. J-SOX does not require auditors to directly assess the effectiveness of companies' internal controls. Instead, auditors must assess the validity of management assessments. That is, auditors must express their opinion regarding whether management reports are accurate based on evidence the auditors gather themselves.

Note 2. Moreover, an audit standard to address risks of fraud in an audit was set in 2013 (Japanese Business Council, 2013).

Note 3. This investigation samples the 2,500 largest companies as measured by the 2012 market value. Only $78 \%$ of CEOs in the U.S. attained their positions through internal promotion. Furthermore, only $25 \%$ of Japanese CEOs worked for another company during their careers compared with 86\% in the U.S. (retrieved April 20, 2017 from http://www.strategyand.pwc.com/media/file/140507_CEO-Data-Media-release-JP.pdf)

Note 4. This survey samples the 84 companies that are members of the Japanese Association of Corporate Directors. (retrieved April 20, 2017 from http://www.jpcd.jp/report/0.041_01_repo.pdf)

Note 5. This study examines companies listed on the TSE on September 10, 2012 (2,275 companies).

Note 6. Including these companies, the chairman of the board is an internal director at $98.8 \%$ of all TSE-listed companies. However, the board chairmen at U.K. companies are barred from having any connection with the CEO position (UK Corporate Governance Code, A.3.1). In the U.S., 20\% of companies in the S\&P 500 Index have an outside chairman, which far exceeds the percentage (12\%) in 2007 (Wall Street Journal Japan, June 12, 2012); this reflects pressure from shareholders.

Note 7. Hennes et al. (2008) distinguish between error and fraud as causes of financial restatements and find that 
financial restatements associated with fraud correlate significantly and positively with executive turnover.

Note 8. Several studies examine the negative consequences of internal control deficiencies. For example, Costello and Wittenberg-Moerman (2011) find that when a firm experiences a material internal control weakness, lenders decrease their use of financial covenants and financial-ratio-based performance pricing provisions and replace them with alternatives. Dhaliwal et al. (2011) find that a firm's credit spread on its publicly traded debt marginally increases if it discloses a material weakness. Moreover, Kim et al. (2011) show that the loan spread is higher for material weaknesses at firms that disclose than for those that do not.

Note 9. This factor shows that consecutive disclosures of material weaknesses are not significantly correlated with $\mathrm{CEO}$ and $\mathrm{CFO}$ turnover.

Note 10. The superiority of a CEO's reputation is measured by whether (a) a CEO who is not serving on one to three boards is replaced by a CEO who is serving on one to three boards; (b) there is no change in CEO; or (c) both the old and new CEOs serve on one to three boards.

Note 11. Cohen et al. (2008) provide four theoretical perspectives (agency theory, resource dependence, institutional theory, and managerial hegemony) to explain the form and substance of corporate governance. Their perspectives are a more comprehensive view of corporate governance than that considered by the traditional agency literature predominately employed in an auditing and accounting studies of governance (Carcello et al., 2011).

Note 12. Moreover, Cohen (2009) illustrates that an audit committee may consist of all independent members (symbolic) but in fact fail to vigorously challenge management over financial reporting quality issues (substantive).

Note 13. The relation between SD disclosure and replacing executives is checked via cross tabulation. The results indicate that executives at companies that have disclosed a SD are replaced substantially more often than executives at companies that have not. Two methods are used to check the endogeneity of SD in this model. The first is the bivariate probit method (which uses Model (1) and the SD model: SD is a dependent variable, and the independent variables are the same as for (1)). The results indicate that H0: $\rho=0$ is not rejected at a significant level (z-value of $\operatorname{atanh} \rho=1.11(\mathrm{p}=0.254)$ ). Second is the instrumental variable method (a probit model with an endogenous regression method); the results indicate that $\mathrm{H} 0: \rho=0$ is not rejected at a significant level. Therefore, SD is treated as an exogenous variable in Model (1).

Note 14. Several previous studies (e.g., Hennes et al. 2008) treat replacing the CFO as a dependent variable. However, few Japanese corporations have a CFO and definitively identifying the CFO is difficult. Therefore, this study considers only CEO replacements.

Note 15. For example, Krishnan (2005) and Hoitash et al. (2009) find that audit committee expertise correlates significantly and negatively with disclosures of material weaknesses in internal controls. Agrawal and Chadha (2005) find that restatements are less likely to occur in companies whose boards or audit committees have an independent director with financial expertise.

Note 16. J-SOX came into force for all listed companies at the end of March 2009. Additionally, from 2012 on, few firms disclosed SDs in Japan (22 firms in 2012, 23 firms in 2013). Therefore, this study's sample period spans 2009-2011.

Note 17. We excluded 108 observations for outlying data identified by Tukey box plotting.

Note 18. The firms that disclosed SDs in correction reports in later years are included in the sample.

Note 19. Although these results restate those in Ge and McVay (2005), the number of Japanese companies that disclose SDs is declining annually across all industries.

Note 20. Corporations disclosing SDs have a relatively high proportion of large shareholders (BIGSHARE, $t=3.02$, $\mathrm{p}<0.01$ ) and low shareholdings by foreign investors (FOREIGNOWN, $\mathrm{t}=-3.62, \mathrm{p}<0.01$ ), banks (BANKING, $\mathrm{t}=$ $-7.26, \mathrm{p}<0.01$ ), and institutional investors (TRUST, $\mathrm{t}=-2.89, \mathrm{p}=0.03$ ).

Note 21. I also created a matched sample based on ROA divided by total assets to ensure the robustness of the regression results. The results of the regression using the matched sample indicate that SD has a positive correlation with TO $(\mathrm{z}=3.516, \mathrm{p}<0.001)$ and $\operatorname{OUTSIDE}(\mathrm{z}=2.192, \mathrm{p}=0.049)$ and has no correlation with EXPERT.

Note 22. Arthaud-Day et al. (2006) argue that restating financials damages organizational legitimacy. Menon and Williams (2008) argue that replacing senior executives signals that directors intend to restore reporting credibility following an auditor resignation.

Note 23. For the 2,437 corporations with fiscal years ending March 31, 2009, regression analysis was performed 
based on the model used in Simunic (1980) and Simunic and Stein (1996). The results showed that corporations disclosing SDs in internal controls (66 corporations) experienced higher audit fees $(\beta=0.322, \mathrm{t}=6.04, \mathrm{p}<0.001)$. This result corresponds with those of Hoitash et al. (2008) and Hogan and Wilkins (2008).

Note 24. These models are based on Hammersley et al. (2012) and Feldmann et al. (2009).

Note 25. Model (6) addresses multicollinearity by including the products of the average deviations of REMEDIATE and TO. Variance inflation factors (VIF) are employed to assess the extent of multicollinearity in Models (5) and (6); the highest VIFs are 1.68 and 1.94, respectively.

Note 26. The Legislative Council of the Ministry of Justice attempted to enact a law requiring the inclusion of one or more outside directors on the corporate boards of listed companies at the June 2012 meeting; however, they shelved the bill after resistance from the business community.

Note 27. See Williamson (2000), Hansmann and Kraakman (2001), and Griffin et al. (2017).

Note 28. See Ohsawa (2010), Sagi (2015), and Hatvany and Pucik (1981).

Note 29. See Yooyanyong and Muenjohn (2010). 